\title{
Formulas for Higher Derivatives of the Riemann Zeta Function
}

\author{
By Tom M. Apostol
}

\begin{abstract}
The functional equation for $\zeta(s)$ is used to obtain formulas for all derivatives $\zeta^{(k)}(s)$. A closed form evaluation of $\zeta^{(k)}(0)$ is given, and numerical values are computed to $15 \mathrm{D}$ for $k=0(1) 18$.
\end{abstract}

The functional equation for the Riemann zeta function states that

$$
\zeta(1-s)=2(2 \pi)^{-s} \cos \frac{\pi s}{2} \Gamma(s) \zeta(s)
$$

(see [3, Theorem 12.7]). If this is differentiated $k$ times we obtain a formula which, as noted by Spira [11], can be put in the form

$$
(-1)^{k} \zeta^{(k)}(1-s)=2(2 \pi)^{-s} \sum_{j=0}^{k} \sum_{m=0}^{k}\left(a_{j k m} \cos \frac{\pi s}{2}+b_{j k m} \sin \frac{\pi s}{2}\right) \Gamma^{(j)}(s) \zeta^{(m)}(s),
$$

where the coefficients $a_{j k m}$ and $b_{j k m}$ are independent of $s$. This formula was used by Spira [11], [12] to determine zero-free regions for $\zeta^{(k)}(s)$, and by Berndt [5], to determine the asymptotic number of zeros of $\zeta^{(k)}(s)$ with $0<t<T$, where $s=\sigma+$ it.

This paper gives a variant of this formula (Theorem 1) which enables us to determine the coefficients $a_{j k m}$ and $b_{j k m}$ explicitly (Theorem 2). Our version also leads to a closed form evaluation of $\zeta^{(k)}(0)$ (Theorem 3) which contains the well-known values $\zeta(0)=-\frac{1}{2}$ and $\zeta^{\prime}(0)=-\frac{1}{2} \log (2 \pi)$ as well as a formula for $\zeta^{\prime \prime}(0)$ obtained by Ramanujan. The results for $k \geqslant 3$ appear to be new. Alternate formulas expressing $\zeta^{(k)}(s)$ in terms of integrals are also given (Theorem 4$)$. The values of $\zeta^{(k)}(0)$ are computed to $16 \mathrm{~S}$ for $k=0(1) 18$ (Table 2).

Notation. Throughout this paper, $z$ denotes the fixed complex number $x+i y$ with $x=-\log 2 \pi, y=-\pi / 2$, and $z^{*}$ denotes the complex conjugate of $z$.

THEOREM 1. For each integer $k \geqslant 1$ and all complex $s$ we have

$$
(-1)^{k} \zeta^{(k)}(1-s)=\sum_{m=0}^{k}\left(\begin{array}{c}
k \\
m
\end{array}\right)\left\{e^{s z} z^{k-m}+e^{s z^{*}}\left(z^{*}\right)^{k-m}\right\}\{\Gamma(s) \zeta(s)\}^{(m)} .
$$

Received January 3, 1984.

1980 Mathematics Subject Classification. Primary $10 \mathrm{H} 05$.

Key words and phrases. Riemann zeta function. 
Proof. First we put the functional equation in a form which is more convenient for repeated differentiation. Since $(2 \pi)^{-s}=e^{-s \log (2 \pi)}$ and $2 \cos \pi s / 2=e^{\pi i s / 2}+e^{-\pi i s / 2}$ we can rewrite (1) as follows:

$$
\zeta(1-s)=\varphi(s ; z)+\varphi\left(s ; z^{*}\right),
$$

where

$$
\varphi(s ; z)=\Gamma(s) \zeta(s) e^{s z}
$$

Differentiation of (3) gives us

$$
(-1)^{k} \zeta^{(k)}(1-s)=\varphi^{(k)}(s ; z)+\varphi^{(k)}\left(s ; z^{*}\right) .
$$

Using Leibniz's rule to calculate $\varphi^{(k)}(s ; z)$ we find

$$
\varphi^{(k)}(s ; z)=e^{s z} \sum_{m=0}^{k}\left(\begin{array}{l}
k \\
m
\end{array}\right) z^{k-m}\{\Gamma(s) \zeta(s)\}^{(m)}
$$

which, together with (4), proves (2).

THEOREM 2. For each integer $k \geqslant 1$ and all complex $s$ we have

$$
\begin{aligned}
& (-1)^{k} \zeta^{(k)}(1-s) \\
& =2(2 \pi)^{-s} \sum_{m=0}^{k}\left(\begin{array}{c}
k \\
m
\end{array}\right)\left\{\operatorname{Re}\left(z^{k-m}\right) \cos \frac{\pi s}{2}\right. \\
& \left.+\operatorname{Im}\left(z^{k-m}\right) \sin \frac{\pi s}{2}\right\}\{\Gamma(s) \zeta(s)\}^{(m)} \\
& =2(2 \pi)^{-s} \sum_{m=0}^{k} \sum_{r=0}^{m}\left(\begin{array}{c}
k \\
m
\end{array}\right)\left(\begin{array}{c}
m \\
r
\end{array}\right)\left\{\operatorname{Re}\left(z^{k-m}\right) \cos \frac{\pi s}{2}\right. \\
& \left.+\operatorname{Im}\left(z^{k-m}\right) \sin \frac{\pi s}{2}\right\} \Gamma^{(r)}(s) \zeta^{(m-r)}(s) .
\end{aligned}
$$

Proof. To deduce (5) from (2) we note that

$$
e^{s z} z^{k-m}+e^{s z^{*}}\left(z^{*}\right)^{k-m}=2(2 \pi)^{-s}\left\{\operatorname{Re}\left(z^{k-m}\right) \cos \frac{\pi s}{2}+\operatorname{Im}\left(z^{k-m}\right) \sin \frac{\pi s}{2}\right\},
$$

and to deduce (6) from (5) we use Leibniz's rule for the $m$ th derivative $\{\Gamma(s) \zeta(s)\}^{(m)}$.

Examples. If $z=x+i y$ we have

$$
\begin{array}{ll}
\operatorname{Re}\left(z^{2}\right)=x^{2}-y^{2}, & \operatorname{Im}\left(z^{2}\right)=2 x y, \\
\operatorname{Re}\left(z^{3}\right)=x^{3}-3 x y^{2}, & \operatorname{Im}\left(z^{3}\right)=3 x^{2} y-y^{3} .
\end{array}
$$

When $x=-\log 2 \pi$ and $y=-\pi / 2$ we find, by taking $k=1,2,3$ in (5),

$$
\begin{aligned}
-\zeta^{\prime}(1-s)= & 2(2 \pi)^{-s}\left\{x \cos \frac{\pi s}{2}+y \sin \frac{\pi s}{2}\right\} \Gamma(s) \zeta(s) \\
& +2(2 \pi)^{-s} \cos \frac{\pi s}{2}\{\Gamma(s) \zeta(s)\}^{\prime}, \\
\zeta^{\prime \prime}(1-s)= & 2(2 \pi)^{-s}\left\{\left(x^{2}-y^{2}\right) \cos \frac{\pi s}{2}+2 x y \sin \frac{\pi s}{2}\right\} \Gamma(s) \zeta(s) \\
& +2(2 \pi)^{-s}\left\{2 x \cos \frac{\pi s}{2}+2 y \sin \frac{\pi s}{2}\right\}\{\Gamma(s) \zeta(s)\}^{\prime} \\
& +2(2 \pi)^{-s} \cos \frac{\pi s}{2}\{\Gamma(s) \zeta(s)\}^{\prime \prime},
\end{aligned}
$$




$$
\begin{aligned}
-\zeta^{\prime \prime \prime}(1-s)= & 2(2 \pi)^{-s}\left\{\left(x^{3}-3 x y^{2}\right) \cos \frac{\pi s}{2}+\left(3 x^{2} y-y^{3}\right) \sin \frac{\pi s}{2}\right\} \Gamma(s) \zeta(s) \\
& +2(2 \pi)^{-s}\left\{3\left(x^{2}-y^{2}\right) \cos \frac{\pi s}{2}+6 x y \sin \frac{\pi s}{2}\right\}\{\Gamma(s) \zeta(s)\}^{\prime} \\
& +2(2 \pi)^{-s}\left\{3 x \cos \frac{\pi s}{2}+3 y \sin \frac{\pi s}{2}\right\}\{\Gamma(s) \zeta(s)\}^{\prime \prime} \\
& +2(2 \pi)^{-s} \cos \frac{\pi s}{2}\{\Gamma(s) \zeta(s)\}^{\prime \prime \prime}
\end{aligned}
$$

It should be noted that when $s$ is an integer one of the factors $\cos \pi s / 2$ or $\sin \pi s / 2$ vanishes, and Eqs. (5) and (6) simplify further. For example, if $s=2 n+1$, where $n=1,2,3, \ldots$, we have $\cos \pi s / 2=0$ and $\sin \pi s / 2=(-1)^{n}$ and (6) becomes

$$
\begin{aligned}
& (-1)^{k} \zeta^{(k)}(-2 n) \\
& \quad=\frac{2(-1)^{n}}{(2 \pi)^{2 n+1}} \sum_{m=0}^{k} \sum_{r=0}^{m}\left(\begin{array}{c}
k \\
m
\end{array}\right)\left(\begin{array}{c}
m \\
r
\end{array}\right) \operatorname{Im}\left(z^{k-m}\right) \Gamma^{(r)}(2 n+1) \zeta^{(m-r)}(2 n+1) .
\end{aligned}
$$

Thus, $\zeta^{(k)}(-2 n)$ is a linear combination of $\zeta(2 n+1), \zeta^{\prime}(2 n+1), \ldots, \zeta^{(k)}(2 n+1)$.

Similarly, when $s=2 n$ the sine terms vanish and we get

$$
(-1)^{k} \zeta^{(k)}(1-2 n)=\frac{2(-1)^{n}}{(2 \pi)^{2 n}} \sum_{m=0}^{k} \sum_{r=0}^{m}\left(\begin{array}{c}
k \\
m
\end{array}\right)\left(\begin{array}{c}
m \\
r
\end{array}\right) \operatorname{Re}\left(z^{k-m}\right) \Gamma^{(r)}(2 n) \zeta^{(m-r)}(2 n)
$$

a linear combination of $\zeta(2 n), \zeta^{\prime}(2 n), \ldots, \zeta^{(k)}(2 n)$.

If we put $s=1$ in (2), we get $(-1)^{k \zeta^{(k)}}(0)$ on the left, but on the right we have an indeterminate form. By expanding each of the functions $e^{s z}, e^{s z^{*}}$ and $\{\Gamma(s) \zeta(s)\}^{(m)}$ in powers of $s-1$ and letting $s \rightarrow 1$ we can obtain a closed form for $(-1)^{k \zeta^{(k)}}(0)$. A simpler method which gives the same result is based on the functional equation in (1).

Since the left member of (1) is analytic at $s=1$ it has a power series expansion

$$
\zeta(1-s)=\sum_{n=0}^{\infty} \frac{(-1)^{n} \zeta^{(n)}(0)}{n !}(s-1)^{n}
$$

Now we expand the right member of (1) in powers of $s-1$ and equate coefficients. Again we use Eq. (3) which served us so well in proving Theorem 1, and first find the expansion of $\varphi(s ; z)$ in powers of $s-1$.

The product $\Gamma(s) \zeta(s)$ has a Laurent expansion of the form

$$
\Gamma(s) \zeta(s)=\frac{1}{s-1}+\sum_{n=0}^{\infty} a_{n}(s-1)^{n},
$$

and for the exponential factor $e^{s z}$ we write

$$
e^{s z}=e^{z} e^{(s-1) z}=\sum_{n=0}^{\infty} e_{n}(z)(s-1)^{n},
$$

where

$$
e_{n}(z)=\frac{e^{z} z^{n}}{n !}
$$


Therefore the product $\varphi(s ; z)=\Gamma(s) \zeta(s) e^{s z}$ has the expansion

$$
\begin{aligned}
\varphi(s ; z) & =\left(\frac{1}{s-1}+\sum_{n=0}^{\infty} a_{n}(s-1)^{n}\right)\left(\sum_{n=0}^{\infty} e_{n}(z)(s-1)^{n}\right) \\
& =\frac{e^{z}}{s-1}+\sum_{n=0}^{\infty}\left(e_{n+1}(z)+\sum_{k=0}^{n} a_{k} e_{n-k}(z)\right)(s-1)^{n}
\end{aligned}
$$

Equating coefficients of $(s-1)^{n}$ in the functional equation (3) we find, for $n \geqslant 0$,

$$
(-1)^{n} \frac{\zeta^{(n)}(0)}{n !}=e_{n+1}(z)+e_{n+1}\left(z^{*}\right)+\sum_{k=0}^{n} a_{k}\left(e_{n-k}(z)+e_{n-k}\left(z^{*}\right)\right)
$$

But $e^{z}=-i /(2 \pi)$ and $e^{z^{*}}=i /(2 \pi)$, so

$$
e_{n}(z)+e_{n}\left(z^{*}\right)=\frac{i z^{n}-i\left(z^{*}\right)^{n}}{2 \pi n !}=\frac{1}{\pi} \frac{\operatorname{Im}\left(z^{n}\right)}{n !}
$$

Hence (8) becomes

$$
(-1)^{n} \frac{\zeta^{(n)}(0)}{n !}=\frac{1}{\pi} \frac{\operatorname{Im}\left(z^{n+1}\right)}{(n+1) !}+\frac{1}{\pi} \sum_{k=0}^{n} a_{k} \frac{\operatorname{Im}\left(z^{n-k}\right)}{(n-k) !} .
$$

Since $\operatorname{Im}\left(z^{0}\right)=0$ and $a_{0}=0$ (as we will show later), the first and last terms of the sum can be deleted and we obtain the following theorem.

THEOREM 3. If $z=-\log (2 \pi)-i \pi / 2$ and $n \geqslant 0$, we have

$$
(-1)^{n} \frac{\zeta^{(n)}(0)}{n !}=\frac{1}{\pi} \frac{\operatorname{Im}\left(z^{n+1}\right)}{(n+1) !}+\frac{1}{\pi} \sum_{k=1}^{n-1} a_{k} \frac{\operatorname{Im}\left(z^{n-k}\right)}{(n-k) !},
$$

where the coefficients $a_{k}$ are determined by (7).

Examples. For $0 \leqslant n \leqslant 4$, we find that (9) gives us

$$
\begin{aligned}
\zeta(0)= & -\frac{1}{2}, \\
\zeta^{\prime}(0)= & -\frac{1}{2 \pi} \operatorname{Im}\left(z^{2}\right)=-\frac{x y}{\pi}=-\frac{1}{2} \log (2 \pi), \\
\zeta^{\prime \prime}(0)= & \frac{1}{3 \pi} \operatorname{Im}\left(z^{3}\right)+\frac{2}{\pi} a_{1} \operatorname{Im}(z)=\frac{1}{3 \pi}\left(3 x^{2} y-y^{3}\right)+\frac{2}{\pi} a_{1} y \\
= & -\frac{1}{2} \log ^{2}(2 \pi)+\frac{\pi^{2}}{24}-a_{1}, \\
\zeta^{\prime \prime \prime}(0)= & -\frac{1}{4 \pi} \operatorname{Im}\left(z^{4}\right)-\frac{3 !}{\pi} \sum_{k=1}^{2} a_{k} \frac{\operatorname{Im}\left(z^{3-k}\right)}{(3-k) !} \\
= & -\frac{1}{2} \log ^{3}(2 \pi)+\frac{\pi^{2}}{8} \log (2 \pi)-3 a_{1} \log (2 \pi)+3 a_{2}, \\
\zeta^{(4)}(0)= & \frac{1}{5 \pi} \operatorname{Im}\left(z^{5}\right)+\frac{4 !}{\pi} \sum_{k=1}^{3} a_{k} \frac{\operatorname{Im}\left(z^{4-k}\right)}{(4-k) !} \\
= & -\frac{1}{2} \log ^{4}(2 \pi)+\frac{\pi^{2}}{4} \log ^{2}(2 \pi)-\frac{\pi^{4}}{160}-6 a_{1} \log ^{2}(2 \pi) \\
& +\frac{\pi^{2}}{2} a_{1}+12 a_{2} \log (2 \pi)-12 a_{3} .
\end{aligned}
$$


The formulas for $\zeta(0)$ and $\zeta^{\prime}(0)$ are well-known [13, p. 20], and the formula for $\zeta^{\prime \prime}(0)$ was obtained by Ramanujan [6, p. 25]. Numerical values are given below in Table 2.

The coefficients $a_{k}$ which appear in (9) and are defined by (7) can be calculated. They are related to the coefficients in the Laurent expansion

$$
\zeta(s+1)=\frac{1}{s}+\sum_{n=0}^{\infty} A_{n} s^{n}
$$

and those in the power series expansion

$$
\Gamma(s+1)=\sum_{n=0}^{\infty} c_{n} s^{n} .
$$

The $A_{n}$ are named after Stieltjes who showed [4, p. 155] that

$$
(-1)^{n} n ! A_{n}=\lim _{N \rightarrow \infty}\left(\sum_{k=1}^{N} \frac{\log ^{n} k}{k}-\frac{\log ^{n+1} N}{n+1}\right) \text {. }
$$

In particular, $A_{0}$ is Euler's constant $\gamma$. The first 20 Stieltjes constants have been calculated by Liang and Todd [9].

The numbers $c_{n}$ in (11) are, of course, $\Gamma^{(n)}(1) / n$ !. The derivatives $\Gamma^{(n)}(1)$ can be expressed in terms of Euler's constant and the values of $\zeta(s)$ at positive integers. This property of the $c_{n}$ is easily derived as follows. Start with the power series expansion for $\psi(x+1)=\Gamma^{\prime}(x+1) / \Gamma(x+1)$, [1, p. 259],

$$
\psi(x+1)=\sum_{n=0}^{\infty}(-1)^{n+1} s_{n+1} x^{n},
$$

where $s_{1}=\gamma$ and $s_{n}=\zeta(n)$ for $n \geqslant 2$. Equating coefficients of $x^{n}$ in the identity $\Gamma^{\prime}(x+1)=\psi(x+1) \Gamma(x+1)$, using (11) and (12), we obtain the recursion formula

$$
(n+1) c_{n+1}=\sum_{k=0}^{n}(-1)^{k+1} s_{k+1} c_{n-k}
$$

with $c_{0}=1$. (See Nielsen [10, p. 40].)

Equation (13) also leads to a closed form evaluation of the derivatives $\Gamma^{(n)}(1)$ in terms of Euler's constant $\gamma$ and $\zeta(2), \zeta(3), \ldots$. For example,

$$
\begin{gathered}
\Gamma^{\prime}(1)=-\gamma, \quad \Gamma^{\prime \prime}(1)=\zeta(2)+\gamma^{2}, \quad \Gamma^{\prime \prime \prime}(1)=-2 \zeta(3)-3 \gamma \zeta(2)-\gamma^{3}, \\
\Gamma^{(4)}(1)=6 \zeta(4)+3 \zeta^{2}(2)+8 \gamma \zeta(3)+6 \gamma^{2} \zeta(2)+\gamma^{4} \\
\Gamma^{(5)}(1)=-24 \zeta(5)-20 \zeta(2) \zeta(3)-15 \gamma \zeta^{2}(2)-30 \gamma \zeta(4) \\
-20 \gamma^{2} \zeta(3)-10 \gamma^{3} \zeta(2)-\gamma^{5} .
\end{gathered}
$$

Jeffery [8] has calculated the first 20 coefficients $c_{n}$ to 12 decimals. Bourguet [7] later calculated to 16 decimals the first 18 coefficients $b_{n}$ in the expansion

$$
(x+1) \Gamma(x+1)=\sum_{n=0}^{\infty} b_{n} x^{n} .
$$

This relation implies $b_{0}=c_{0}=1$ and

$$
b_{n}=c_{n}+c_{n-1} \text { for } n \geqslant 1,
$$


TABLE 1

\begin{tabular}{rrr}
\hline$n$ & Stieltjes constants $A_{n}$ & $c_{n}=\Gamma^{(n)}(1) / n !$ \\
\hline 0 & 0.5772156649015329 & 1.000000000000000000 \\
1 & $0.7281584548367672(-01)$ & -0.5772156649015329 \\
2 & $-0.4845181596436160(-02)$ & 0.9890559953279726 \\
3 & $-0.3423057367172240(-03)$ & -0.9074790760808863 \\
4 & $0.9689041939447080(-04)$ & 0.9817280868344002 \\
5 & $-0.6611031810842190(-05)$ & -0.9819950689031452 \\
6 & $0.3316240908752770(-06)$ & 0.9931491146212762 \\
7 & $0.1046209458447920(-06)$ & -0.9960017604424315 \\
8 & $-0.8733218100273800(-08)$ & 0.9981056937831289 \\
9 & $0.9478277782762000(-10)$ & -0.9990252676219549 \\
10 & $0.5658421927608700(-10)$ & 0.9995156560727774 \\
11 & $-0.6768689863514000(-11)$ & -0.9997565975086013 \\
12 & $0.3492115936670000(-12)$ & 0.9998782713151333 \\
13 & $0.4410424742000000(-14)$ & -0.9999390642064443 \\
14 & $-0.2399786222000000(-14)$ & 0.9999695177634821 \\
15 & $0.2167731220000000(-15)$ & -0.9999847526993770 \\
16 & $-0.9544466000000000(-17)$ & 0.9999923744790732 \\
17 & $-0.7387700000000000(-19)$ & -0.9999961865894733 \\
18 & $0.4800900000000000(-19)$ & 0.9999980930811309 \\
& & -0.999999046469 \\
& & \\
& &
\end{tabular}

TABLE 2

\begin{tabular}{rrll}
\hline$n$ & \multicolumn{1}{c}{$a_{n}$} & \multicolumn{1}{c}{$\zeta^{(n)}(0)$} & \multicolumn{1}{c}{$\zeta^{(n)}(0) / n !$} \\
\hline 0 & 0.0000000000000000 & -0.5000000000000000 & -0.5000000000000000 \\
1 & 0.7286939170039305 & -0.9189385332046727 & -0.9189385332046727 \\
2 & -0.3834560903754670 & -2.006356455908585 & -1.003178227954292 \\
3 & 0.5323903060606865 & -6.004711166862254 & -1.000785194477042 \\
4 & -0.4859027759456871 & -23.99710318801370 & -0.9998792995005709 \\
5 & 0.5018073423500181 & -120.0002329075584 & -1.000001940896320 \\
6 & -0.4985920362510443 & -720.0009368251297 & -1.000001301146014 \\
7 & 0.4998425924690323 & -5039.999150176233 & -0.9999998313841731 \\
8 & -0.4998028591976903 & -40320.00023243172 & -1.000000005764676 \\
9 & 0.4999251541081416 & -362880.0003305895 & -1.000000000911016 \\
10 & -0.4999581497598492 & -3628799.999456764 & -0.9999999998502988 \\
11 & 0.4999798488252394 & -39916800.00037562 & -1.000000000009410 \\
12 & -0.4999897969263561 & -479001600.0000220 & -1.000000000000046 \\
13 & 0.4999949183147713 & -6227020799.999629 & -0.9999999999999405 \\
14 & -0.4999974562188593 & -87178291200.00114 & -1.000000000000013 \\
15 & 0.4999987285230217 & -1307674368000.008 & -1.000000000000006 \\
16 & -0.4999993642057035 & -20922789888000.15 & -1.000000000000007 \\
17 & 0.4999996821100205 & -355687428096002.6 & -1.000000000000007 \\
18 & -0.49999984106 & -6402373705728048. & -1.000000000000008 \\
\hline
\end{tabular}

so we have a simple way of calculating the $c_{n}$ in (11) recursively from the $b_{n}$. The numerical values of the $c_{n}$ in Table 1 were obtained in this way from Bourguet's values. When rounded off to 12 decimals they agree with Jeffery's results except for $c_{10}$ where Jeffery lists the 12 th decimal place as 4 instead of 2 .

To relate $a_{n}$ to the $A_{n}$ and $c_{n}$ we write (7) as

$$
\Gamma(s+1) \zeta(s+1)=\frac{1}{s}+\sum_{n=0}^{\infty} a_{n} s^{n},
$$


then multiply (10) and (11), and equate coefficients to get

$$
a_{n}=c_{n+1}+\sum_{k=0}^{n} A_{k} c_{n-k} .
$$

This gives a closed form evaluation of the $a_{n}$ in terms of the Stieltjes constants and values of the zeta function at positive integers. The first few values are

$$
\begin{aligned}
& a_{0}=c_{1}+A_{0}=0, \\
& a_{1}=\frac{1}{2} \zeta(2)-\frac{1}{2} \gamma^{2}+A_{1}, \\
& a_{2}=-\frac{1}{3} \zeta(3)+\frac{1}{3} \gamma^{3}-\gamma A_{1}+A_{2}, \\
& a_{3}=\frac{1}{4} \zeta(4)+\frac{1}{8} \zeta^{2}(2)-\frac{1}{4} \gamma^{2} \zeta(2)-\frac{1}{8} \gamma^{4}+\frac{1}{2} \zeta(2) A_{1}+\frac{1}{2} \gamma^{2} A_{1}-\gamma A_{2}+A_{3} .
\end{aligned}
$$

Numerical values for the $a_{n}$ are given in Table 2. The calculations were based on (14) using the values for the $A_{n}$ given by Liang and Todd [9] and the values of $c_{n}$ listed in Table 1. These values, in turn, were used together with (9) to calculate the derivatives $\zeta^{(n)}(0)$ in Table 2 . The numbers in Table 2 reveal that $(-1)^{n} a_{n}$ converges to $-\frac{1}{2}$ and that $\zeta^{(n)}(0) / n$ ! converges to -1 . These facts are easily proved by observing that we have the power series expansions

$$
\zeta(1-s)+\frac{1}{s}=\sum_{n=0}^{\infty}\left(\frac{\zeta^{(n)}(0)}{n !}+1\right)(1-s)^{n}
$$

and

$$
\Gamma(s) \zeta(s)+\frac{1}{2 s}=\sum_{n=0}^{\infty}\left((-1)^{n} a_{n}+\frac{1}{2}\right)(1-s)^{n},
$$

each of which converges for $s=0$; so when $s=0$ the general term of each series tends to 0 .

Alternate formulas for $\zeta^{(k)}(s)$ can be obtained from the representation [1, p. 807]

$$
\zeta(s)=\frac{1}{s-1}+\frac{1}{2}+\sum_{r=1}^{n} \frac{B_{2 r}}{2 r}\left(\begin{array}{c}
s+2 r-2 \\
2 r-1
\end{array}\right)-\left(\begin{array}{c}
s+2 n \\
2 n+1
\end{array}\right) \int_{1}^{\infty} \frac{P_{2 n+1}(x)}{x^{s+2 n+1}} d x
$$

which is a consequence of Euler's summation formula. The $B_{2 r}$ are Bernoulli numbers and the integral involves the periodic Bernoulli function

$$
P_{2 n+1}(x)=(-1)^{n+1} \frac{2(2 n+1) !}{(2 \pi)^{2 n+1}} \sum_{k=1}^{\infty} \frac{\sin 2 k \pi x}{k^{2 n+1}} .
$$

The representation for $\zeta(s)$ in (15) is valid in the half-plane $\sigma>-2 n, n=1,2,3, \ldots$, and can be rewritten as follows:

$$
\zeta(s)=\frac{1}{s-1}+\frac{1}{2}+\sum_{r=1}^{n} \frac{B_{2 r}}{2 r} Q_{2 r-1}(s)-Q_{2 n+1}(s) I_{2 n+1}(s),
$$

where $Q_{m}(s)$ is the binomial coefficient (a polynomial in $s$ of degree $m$ ),

$$
Q_{m}(s)=(s+\underset{m}{m}-1)=\frac{s(s+1)(s+2) \cdots(s+m-1)}{m !}
$$

and

$$
I_{m}(s)=\int_{1}^{\infty} \frac{P_{m}(x)}{x^{s+m}} d x
$$


The $k$ th derivative of this integral is simply

$$
I_{m}^{(k)}(s)=(-1)^{k} \int_{1}^{\infty} \frac{P_{m}(x)(\log x)^{k}}{x^{s+m}} d x
$$

and the $k$ th derivative of $Q_{m}(s)$ vanishes identically if $k>m$.

By differentiating (17) repeatedly we obtain the following theorem.

THEOREM 4. In the half-plane $\sigma>-2 n, n=1,2,3, \ldots$, we have

$$
\begin{aligned}
\zeta^{(k)}(s)= & \frac{(-1)^{k} k !}{(s-1)^{k+1}}+\sum_{r=1}^{n} \frac{B_{2 r}}{2 r} Q_{2 r-1}^{(k)}(s) \\
& -\sum_{\nu=0}^{k}\left(\begin{array}{l}
k \\
\nu
\end{array}\right) Q_{2 n+1}^{(\nu)}(s) I_{2 n+1}^{(k-\nu)}(s) .
\end{aligned}
$$

For the special case $n=1$ the sum on $r$ vanishes identically if $k \geqslant 2$, and the sum on $\nu$ contains at most four terms since $Q_{3}^{(\nu)}(s)$ vanishes identically for $\nu>3$. Thus, for $\sigma>-2$ we have

$$
\zeta^{\prime}(s)=\frac{-1}{(s-1)^{2}}+\frac{1}{12}-\frac{s(s+1)(s+2)}{6} I_{3}^{\prime}(s)-\frac{3 s^{2}+6 s+2}{6} I_{3}(s)
$$

and, for $k \geqslant 2$,

$$
\begin{aligned}
\zeta^{(k)}(s)= & \frac{(-1)^{k} k !}{(s-1)^{k+1}}-\frac{s(s+1)(s+2)}{6} I_{3}^{(k)}(s) \\
& -\frac{k}{6}\left(3 s^{2}+6 s+2\right) I_{3}^{(k-1)}(s)-\frac{k(k-1)}{2}(s+1) I_{3}^{(k-2)}(s) \\
& -k(k-1)(k-2) I_{3}^{(k-3)}(s) .
\end{aligned}
$$

(If $k=2$ the last term on the right of (22) is understood to be zero.)

When $s=0$ the formulas are even simpler. From (21) we get

$$
\zeta^{\prime}(0)=-1+\frac{1}{12}-\frac{1}{3} I_{3}(0)
$$

and for $k \geqslant 2$, we have

$$
\begin{aligned}
\zeta^{(k)}(0)= & -k !-\frac{k}{3} I^{(k-1)}(0)-\frac{k(k-1)}{2} I_{3}^{(k-2)}(0) \\
& -k(k-1)(k-2) I_{3}^{(k-3)}(0) .
\end{aligned}
$$

These formulas, used in conjunction with Theorem 3 , lead to successive closed form evaluations of the integrals $I_{3}(0), I_{3}^{\prime}(0), I_{3}^{\prime \prime}(0), \ldots$. For example, using the formulas derived earlier for $\zeta^{\prime}(0)$ and $\zeta^{\prime \prime}(0)$, we find

$$
I_{3}(0)=\int_{1}^{\infty} \frac{P_{3}(x)}{x^{3}} d x=-\frac{11}{4}+\frac{3}{2} \log (2 \pi)=0.006815599614018225
$$

and

$$
\begin{aligned}
I_{3}^{\prime}(0) & =\int_{1}^{\infty} \frac{P_{3}(x)(-\log x)}{x^{3}} d x \\
& =\frac{9}{8}-\frac{9}{4} \log (2 \pi)+\frac{3}{4} \log ^{2}(2 \pi)-\frac{\pi^{2}}{16}+\frac{3}{2} a_{1}=-0.000688715558150 .
\end{aligned}
$$


The same type of analysis can be applied to the Hurwitz zeta function $\zeta(s, a)$, the analytic continuation of the series

$$
\zeta(s, a)=\sum_{n=0}^{\infty} \frac{1}{(n+a)^{s}}
$$

where $a>0$ and $\sigma>1$. For $\sigma>-1$, Euler's summation formula gives the representation

$$
\zeta(s, a)=a^{-s}\left(\frac{1}{2}+\frac{a}{s-1}\right)-s(s+1) \int_{0}^{\infty} \frac{\varphi_{2}(x)}{(x+a)^{s+2}} d x,
$$

where $\varphi_{2}(x)=\int_{0}^{x}\left(t-[t]-\frac{1}{2}\right) d t$ is periodic with period 1 and satisfies

$$
\varphi_{2}(x)=\frac{1}{2} x(x-1) \text { if } 0 \leqslant x \leqslant 1 .
$$

Differentiating (25) $k$ times we obtain the formula

$$
\begin{aligned}
(-1)^{k} \zeta^{(k)}(s, a)= & \left(\log ^{k} a\right) a^{-s}\left(\frac{1}{2}+\frac{a}{s-1}\right)+k ! a^{1-s} \sum_{r=0}^{k-1} \frac{\log ^{r} a}{r !(s-1)^{k-r+1}} \\
& -s(s+1) \int_{0}^{\infty} \frac{\varphi_{2}(x) \log ^{k}(x+a)}{(x+a)^{s+2}} d x \\
& +k(2 s+1) \int_{0}^{\infty} \frac{\varphi_{2}(x) \log ^{k-1}(x+a)}{(x+a)^{s+2}} d x \\
& -k(k-1) \int_{0}^{\infty} \frac{\varphi_{2}(x) \log ^{k-2}(x+a)}{(x+a)^{s+2}} d x .
\end{aligned}
$$

For $s=0$ this simplifies to

$$
\begin{aligned}
\zeta^{(k)}(0, a)= & \left(\log ^{k} \frac{1}{a}\right)\left(\frac{1}{2}-a\right)-k !+k ! a \sum_{r=k}^{\infty} \frac{\log ^{r} 1 / a}{r !} \\
& +(-1)^{k} k \int_{0}^{\infty} \frac{\varphi_{2}(x) \log ^{k-1}(x+a)}{(x+a)^{2}} d x \\
& -(-1)^{k} k(k-1) \int_{0}^{\infty} \frac{\varphi_{2}(x) \log ^{k-2}(x+a)}{(x+a)^{2}} d x .
\end{aligned}
$$

When $a=1$ this can be transformed to (24) using integration by parts.

When $a=1$ and $k=1$, Eq. (26) reduces to

$$
\zeta^{\prime}(0)=-1-\int_{0}^{\infty} \frac{\varphi_{2}(x)}{(x+1)^{2}} d x=-1-\int_{1}^{\infty} \frac{\varphi_{2}(x)}{x^{2}} d x .
$$

But, from [2, p. 616], we see that

$$
1+\int_{1}^{\infty} \frac{\varphi_{2}(x)}{x^{2}} d x=\frac{1}{2} \log (2 \pi)
$$

so we have another derivation of the formula

$$
\zeta^{\prime}(0)=-\frac{1}{2} \log (2 \pi) \text {. }
$$

Alfred P. Sloan Laboratory of Mathematics and Physics California Institute of Technology

Pasadena, California 91125 
1. Milton Abramowitz \& Irene A. Stegun, Handbook of Mathematical Functions, Nat. Bur. Standards, Appl. Math. Series No. 55, Washington, D. C., 1964.

2. Том M. Apostol, Calculus, Vol. II, 2nd ed., Wiley, New York, 1969.

3. Tом M. APOSTOL, Introduction to Analytic Number Theory, Undergraduate Texts in Mathematics, Springer-Verlag, New York, 1976.

4. B. Baillaud \& H. Bourget, Correspondence d'Hermite et de Stieltjes, Tome I, Gauthier-Villars, Paris, 1905.

5. BRuce C. Berndt, “The number of zeros for $\zeta^{(k)}(s)$," J. London Math. Soc. (2), v. 2, 1970, pp. 577-580.

6. BRUCE C. Berndt, “Chapter 8 of Ramanujan's second notebook," J. Reine Angew. Math., v. 338, 1983, pp. 1-55.

7. L. Bourguet, "Sur les intégrales Euleriennes et quelques autres fonctions uniformes," Acta Math., Bd. 2, 1883, pp. 261-295.

8. HenRY M. JefFery, “On the derivatives of the gamma-function,” Quart. J. Math., v. 6, 1864, pp 82-108.

9. J. J. Y. Liang \& JoHN TOdD, “The Stieltjes constants,” J. Res. Nat. Bur. Standards Sect. B, v. 76B, 1972, pp. 161-178.

10. Niels NiElSEN, Handbuch der Theorie der Gammafunktion, Teubner, Leipzig, 1906.

11. Robert SpIRA, “Zero-free regions of $\zeta^{(k)}(s)$," J. London Math. Soc., v. 40, 1965, pp. 677-682.

12. Robert SPIRA, “Another zero-free region for $\zeta^{(k)}(s)$," Proc. Amer. Math. Soc., v. 26, 1970, pp. 246-247.

13. E. C. Titchmarsh, The Theory of the Riemann Zeta-Function, Clarendon Press, Oxford, 1951. 